\title{
Aplastic anaemia and reticulated skin hyperpigmentation
}

\author{
Halûk Demiroğlu, Mehmet Alikaşifoğlu, Başak Yalçın, Semra Dündar
}

A 32-year-old man was hospitalised for pancytopenia in August 1995. He had been complaining of increasing fatigue for the last six months. He had reticulated skin hyperpigmentation, most noticeable on the neck and thorax (figure). He had first noticed the skin changes when he was 10 years old and lost his fingernails at the age of 13. He also complained of epiphora. His parents' marriage was not consanguinous and both his parents, two sisters, a brother and his three-yearold son were normal in health.

On ophthalmic examination, obliteration of punctae lacrimalis was observed. Leukoplakia was present on the tongue. Hepatosplenomegaly and lymphadenopathy could not be detected. On admission, investigations revealed a haemoglobin of $7.3 \mathrm{~g} / \mathrm{dl}$, white blood cell count $1.8 \times 10^{9} / 1$ and platelets $32 \times 10^{\%} /$. Peripheral blood film showed macrocytic red cells with a differential of $41 \%$ neuthrophils, $52 \%$ lymphocytes, $6 \%$ monocytes and $1 \%$ eosinophils. Biochemical analysis of the blood was within the normal range as were the immunoglobulin levels. No pathology was detected in skeletal films and intravenous pyelography. The bone marrow biopsy showed extreme hypocellularity with an increase in fat droplets, and reticulin staining did not show signs of myelofibrosis.

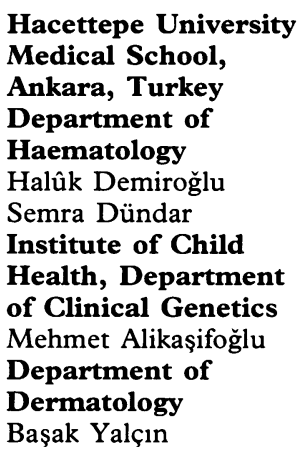

Correspondence to Halûk Demiroğlu, MD, Hoşdere Cad. $80 / 19$, Yukarı Ayranc1, 06550, AnkaraTURKEY

Accepted 22 January 1997

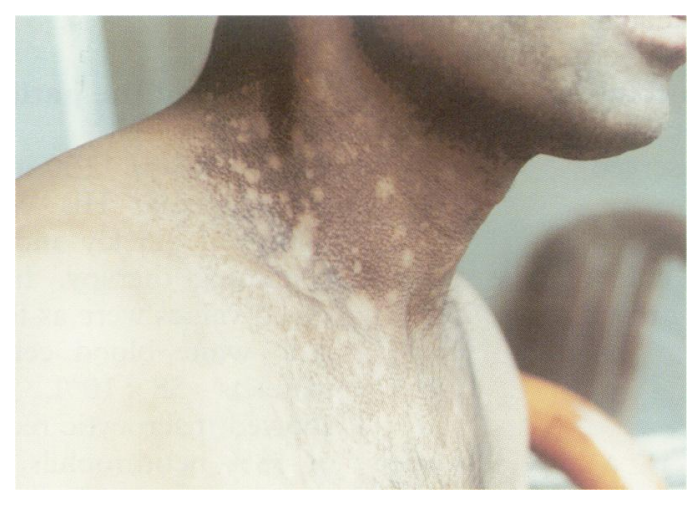

Figure Reticular hyperpigmentation on the neck and thorax

\section{Questions}

1 What is the diagnosis?

2 What kind of histopathologic changes does skin biopsy of the lesion reveal?

3 What disease is most commonly confused with this disorder and how is the differential diagnosis made?

4 What are the serious/fatal complications of the disease? 


\section{Answers \\ QUESTION 1 \\ Dyskeratosis congenita.}

\section{QUESTION 2}

Histopathologic examination of reticularly pigmented skin generally shows hyperkeratosis, epidermal atrophy and liquefaction degeneration of basal layer. ${ }^{1}$

\section{QUESTION 3}

Dyskeratosis congenita has many features in common with another inherited bone marrow failure syndrome, Fanconi's anaemia. ${ }^{2}$ Fanconi's anaemia also causes skin pigmentation, although this is more uniform. Renal and limb reduction anomalies are commonly found in Fanconi's anaemia, but not in dyskeratosis congenita. Genetic studies may help establish a differential diagnosis. Both dyskeratosis congenita and Fanconi's anaemia are chromosomal instability disorders. However, in dyskeratosis congenita, clastogen-induced sister chromatid exchange frequency is increased, in contrast to Fanconi's anaemia in which it is normal. ${ }^{2}$ When peripheral blood lymphocytes from patients with Fanconi's anaemia are induced with diepoxybutane, chromosomal breaks are not usually observed. ${ }^{3}$

\section{QUESTION 4}

Pancytopenia and malignancy are the serious and fatal complications of this disease. ${ }^{4}$

\section{Discussion}

Dyskeratosis congenita is a rare disorder characterised by the triad of reticulated skin hyperpigmentation, nail dystrophy and leukoplakia of mucous membranes. ${ }^{1,2}$ Cutaneous findings are the most consistent feature of dyskeratosis congenita. Reticulated skin pigmentation most commonly affects the face, neck, chest and arms. The degree of pigmentation increases with age and can involve the entire skin surface. There is also a high association of haematopoietic abnormalities

1 Singh K, Kolalapudi S. Dyskeratosis congenita: report of the first case from India. $\mathcal{F}$ Dermatol 1986; 13: 54-8.

2 Dokal I. Dyskeratosis congenita: an inherited bone marrow failure syndrome. $\operatorname{Br} \mathcal{F}$ Haematol 1996; 92: 775 -9.

3 Auerbach AD, Rogatko A, Schoreder-Kurth TM. International Fanconi Anemia Registry: Relation of clinical symptoms to diepoxybutane sensitivity. Blood 1989; 73: $391-6$. with this syndrome. The earliest sign may be anaemia or thrombocytopenia, but ultimate progression to aplastic anaemia is usual. Patients are prone to develop secondary cutaneous and mucosal carcinomas. ${ }^{4}$ Less common manifestations of the disease are mental retardation, small stature, deafness, epiphora and oral/dental abnormalities. ${ }^{5}$

Pancytopenia with a hypocellular bone marrow is one of the serious complications of dyskeratosis congenita. The reason for progressive bone marrow failure is currently unknown. While genetic abnormalities are unusual in acquired aplastic anaemia, chromosomal changes are common in constitutional forms of the disease. It is possible that chromosome instability leads to progressive depletion of the stem cell pool which manifests as pancytopenia. ${ }^{2,5}$ The primary defect responsible for this disease is unknown. Variable frequencies of chromosomal abnormalities suggest that a defect in DNA repair that might be of fundamental importance, both in the aetiology of dyskeratosis congenita and the increased incidence of neoplasia in these patients. ${ }^{2,4,5}$

\section{Progress of the patient}

Oxymethalone $150 \mathrm{mg} /$ day was begun for pancytopenia. His constitutional symptoms improved quickly and he felt quite well after beginning therapy. In November 1996, his blood values were as follows: haemoglobin 9.1 $\mathrm{g} / \mathrm{dl}$, white blood cell count $2.7 \times 10^{9} / 1$ and platelets $58 \times 10^{9} / 1$. Peripheral blood film showed macrocytic red cells with a differential of $58 \%$ neuthrophils, $36 \%$ lymphocytes, $5 \%$ monocytes and $1 \%$ eosinophil (note reversal of neutrophil/lymphocyte ratio).

\section{Final diagnosis}

Dyskeratosis congenita.

Keywords: dyskeratosis congenita, aplastic anemia, chromosomal instability

4 Davidson HR, Connor JM. Dyskeratosis congenita. $\mathcal{f ~ M e d}$ Genet 1988; 25: 843-6.

5 Sirinavin C, Trowbridge AA. Dyskeratosis congenita: clinical features and genetic aspects. Report of a family and review of the literature. $\mathcal{F}$ Med Genet 1975; 12: 339-54 